\title{
C-Myc Overexpression Increases Ribosome Biogenesis and Protein Synthesis Independent of mTORC1 Activation in Mouse Skeletal Muscle
}

\section{Takahiro Mori}

Nagoya Institute of Technology

\section{Satoru Ato}

Nagoya Institute of Technology

Jonas R. Knudsen

University of Copenhagen

Carlos Henriquez-Olguin

University of Copenhagen

\section{Zhencheng Li}

University of Copenhagen

\section{Koki Wakabayashi}

Nagoya Institute of Technology

\section{Takeshi Suginohara}

Nagoya Institute of Technology

Kazuhiko Higashida

University of Shiga Prefecture

Yuki Tamura

Nippon Sport Science University

Koichi Nakazato

Nippon Sport Science University

Thomas E. Jensen

University of Copenhagen

Riki Ogasawara ( $\square$ ogasawara.riki@nitech.ac.jp )

Nagoya Institute of Technology https://orcid.org/0000-0002-2600-1613

\section{Research}

Keywords: exercise, c-Myc, ribosome biogenesis, protein metabolism, RNA-Seq

Posted Date: December 14th, 2020

DOI: https://doi.org/10.21203/rs.3.rs-124889/v1 
License: (c) (i) This work is licensed under a Creative Commons Attribution 4.0 International License. Read Full License 


\section{c-Myc overexpression increases ribosome biogenesis and protein synthesis independent of mTORC1 activation in mouse skeletal muscle}

Takahiro Mori ${ }^{1}$, Satoru Ato ${ }^{1}$, Jonas R. Knudsen ${ }^{2,3}$, Carlos Henriquez-Olguin ${ }^{2}$, Zhencheng Li $^{2}$, Koki Wakabayashi ${ }^{1}$, Takeshi Suginohara ${ }^{1}$, Kazuhiko Higashida ${ }^{4}$, Yuki Tamura ${ }^{5}$, Koichi Nakazato ${ }^{5}$, Thomas E. Jensen ${ }^{2, *}$, Riki Ogasawara ${ }^{1,2, *}$

${ }^{1}$ Department of Life Science and Applied Chemistry, Nagoya Institute of Technology, Nagoya, Japan ${ }^{2}$ Section of Molecular Physiology, Department of Nutrition, Exercise and Sports, University of Copenhagen, Denmark.

${ }^{3}$ Microsystems Laboratory 2, Institute of Microengineering, École Polytechnique Fédérale de Lausanne, Switzerland

${ }^{4}$ Department of Nutrition, University of Shiga Prefecture, Hikone, Japan

${ }^{5}$ Graduate School of Health and Sport Science, Nippon Sport Science University, Tokyo, Japan

*Corresponding authors:

Riki Ogasawara, Ph.D

E-mail: ogasawara.riki@nitech.ac.jp

Thomas E. Jensen, Ph.D

E-mail: tejensen@nexs.ku.dk

Running title: c-Myc stimulates ribosome biogenesis and protein synthesis 


\begin{abstract}
Background: High-intensity muscle contractions (HiMC) are known to increase c-Myc expression which is known to stimulate ribosome biogenesis and protein synthesis in tumor cells. However, whether the increase in c-Myc stimulates ribosome biogenesis and protein synthesis in skeletal muscles remains unknown.
\end{abstract}

Methods: We investigated the effect of adeno-associated virus (AAV)-mediated c-Myc overexpression, with or without fasting or percutaneous electrical stimulation-induced HiMC, on ribosome biogenesis and protein synthesis in adult mouse skeletal muscles.

Results: AAV-mediated overexpression of c-Myc in mouse skeletal muscles for 2 weeks increased the DNA polymerase subunit POL1 mRNA, 45S-pre-rRNA, total RNA, and muscle protein synthesis without altering mechanistic target of rapamycin complex 1 (mTORC1) signaling under both ad libitum and fasted conditions. RNA-seq analyses revealed that c-Myc overexpression mainly regulated ribosome biogenesis-related biological processes. The protein synthesis response to c-Myc overexpression mirrored the response with HiMC. No additional effect of combining c-Myc overexpression and HiMC was observed.

Conclusion: c-Myc overexpression is sufficient to stimulate skeletal muscle ribosome biogenesis and protein synthesis without activation of mTORC1. Therefore, the HiMC-induced increase in c-Myc may contribute to ribosome biogenesis and increased protein synthesis following HiMC.

Keywords: exercise, c-Myc, ribosome biogenesis, protein metabolism, RNA-Seq 


\section{Background}

High-intensity muscle contraction (HiMC)-stimulated protein synthesis, such as after resistance exercise, is believed to contribute to increases in skeletal muscle mass over time. Acutely, HiMC stimulates muscle protein synthesis by facilitating translational efficiency while more chronic adaptations include an increase in basal muscle protein synthesis by increasing ribosome availability/translational capacity (1-3). Simultaneously, muscle protein degradation remains unchanged or increases slightly after resistance exercise $(4,5)$. Therefore, HiMC-induced net positive protein balance is thought to be mainly due to increased muscle protein synthesis rather than decreased muscle protein degradation.

Mechanistic target of rapamycin (mTOR), especially mTOR complex 1 (mTORC1), is a major regulator of protein synthesis by regulating both translational efficiency and capacity $(6,7)$. However, recent studies have indicated that muscle contraction-induced increase in protein synthesis is not solely dependent on mTORC1 (8-13). The transcription factor c-Myc is also known to stimulate protein synthesis in tumor cells, possibly by stimulating ribosome biogenesis $(14,15)$. Previous studies reported that HiMC acutely increased c-Myc expression at both mRNA and protein levels $(8,11,16-18)$. The increase in c-Myc after HiMC was also observed during treatment with the mTORC1 inhibitor, rapamycin $(8,11)$. Moreover, an increase in c-Myc protein was observed after chronic resistance training in rats and humans $(8,19,20)$. Therefore, resistance exercise-induced increase in c-Myc could contribute to ribosome biogenesis and protein synthesis. In support of this, pharmacological inhibition of c-Myc in $\mathrm{C} 2 \mathrm{C} 12$ myotubes inhibited protein synthesis (11). Moreover, a recent study reported that c-Myc overexpression in the skeletal muscle of 1-day-old chick induced muscle hypertrophy (21). However, whether c-Myc upregulation induces ribosome biogenesis and protein synthesis in mature skeletal muscle in vivo remains unclear.

In addition to its role in the transcriptional regulation of ribosome biogenesis, c-Myc regulates transcription of several genes linked to cell growth and metabolism in a B lymphoid tumor model (22, 23 ), indicating that many of the HiMC-induced changes in skeletal muscle gene expression could be mediated by c-Myc upregulation. However, whether this is the case is presently unknown. Thus, we investigated the effect of c-Myc overexpression on gene expression, ribosome biogenesis, and protein synthesis in skeletal muscle in this study. Further, we investigated how c-Myc overexpression interacted with suppression and stimulation of mTORC1 signaling and protein synthesis during fasting and after HiMC. 


\section{Methods}

\section{Ethical approval}

All experimental procedures performed in this study were approved by the Danish Animal Experimental Inspectorate (License \# 2017-15-0201-01311) and carried out in accordance with the European Convention for Vertebrate Animals Used for Experiments and Other Scientific Purposes.

\section{Animals}

Female C57BL/6J mice (11 weeks old) purchased from Taconic (Lille Skensved, Denmark) were housed under $12 \mathrm{~h}$ light/dark cycle, with food and water ad libitum.

\section{Adeno-associated virus vectors}

Adeno-associated virus serotype 6 (AAV6) that express mouse c-Myc (NM_001177352) under the control of the CMV promoter (AAV6-c-Myc) or empty control CMV vector (AAV6-CON) were generated, and $1 \times 10^{10}$ vector genomes were injected into the right and left gastrocnemius muscles of mice, respectively, under $2 \%$ isoflurane anesthesia.

\section{Experiment 1: c-Myc overexpression during fasting}

Two weeks after AAV6-c-Myc and AAV6-CON injection, mice were fasted from 8 AM. Muscle samples were obtained after cervical dislocation before, $3 \mathrm{~h}$, and $9 \mathrm{~h}$ after food removal. The muscles were frozen rapidly in liquid nitrogen and stored at $-80^{\circ} \mathrm{C}$ until further analyses.

\section{Experiment 2: c-Myc overexpression on HiMC effects}

Two weeks after AAV6-c-Myc and AAV6-CON injection, the mice were anesthetized with 2\% isoflurane, and the right gastrocnemius muscle was contracted isometrically by fixing the sole of the foot at a $90^{\circ}$ angle using percutaneous electrical stimulation $(100 \mathrm{~Hz}, 5$ sets of ten $3 \mathrm{~s}$ contractions, $7 \mathrm{~s}$ rest between contractions, 3 min rest between sets), as described previously (24). The left gastrocnemius muscle served as a control. Muscle samples were obtained after cervical dislocation $3 \mathrm{~h}$ after muscle contraction. Tissues were frozen rapidly in liquid nitrogen and stored at $-80^{\circ} \mathrm{C}$ until further analyses.

\section{Western blotting}

Powdered frozen muscle samples were homogenized in 10 times the volume of lysis buffer $(20 \mathrm{mM}$ Tris-HCl [pH 7.5], 1\% NP40, 1\% sodium deoxycholate, $1 \mathrm{mM}$ EDTA, $1 \mathrm{mM}$ EGTA, and $150 \mathrm{mM}$ $\mathrm{NaCl}$ ) containing Halt ${ }^{\mathrm{TM}}$ protease and phosphatase inhibitor cocktail (Thermo Fisher Scientific, Waltham, MA, USA). After centrifugation at $10,000 \times g$ for $10 \mathrm{~min}$ at $4{ }^{\circ} \mathrm{C}$, the supernatant was collected and the protein concentration of each sample was determined using a Protein Assay Rapid Kit Wako II (FUJIFILM Wako, Osaka, Japan). Samples were diluted in lysis buffer to equal protein concentration, mixed with $3 \times$ sample buffer, and boiled at $95^{\circ} \mathrm{C}$ for 5 min. Equal amounts of protein 
were separated electrophoretically and subsequently semi-dry transferred to ClearTrans ${ }^{\circledR}$ SP polyvinylidene difluoride (PVDF) membranes (FUJIFILM). The membranes were washed in Trisbuffered saline containing $0.1 \%$ Tween-20 (TBST) and blocked with $1 \%$ dry milk for 60 min at 22$24^{\circ} \mathrm{C}$. The membranes were then washed and incubated overnight at $4^{\circ} \mathrm{C}$ with specific primary antibodies. Antibodies against phospho-p70S6K (Thr389, cat\#9205), total-p70S6K (cat\#2708), phospho-4E-BP1 (Thr36/45, cat\#9459), total-4E-BP1 (cat\#9452), phospho-AMPK (Thr172, cat\#2535), total-AMPK (cat\#2532), LC3B (cat\#2775), ubiquitin (cat\#3933), and c-Myc (cat\#5605) were obtained from Cell Signaling Technology (Danvers, MA, USA). Antibodies against total-REDD1 (cat\#10638-1AP) was obtained from Proteintech (Rosemont, IL, USA). Antibodies against p62 (cat\#PM045) were obtained from MBL (Nagoya, Japan). The membranes were washed again in TBST and incubated for $1 \mathrm{~h}$ at room temperature with an anti-rabbit secondary antibody (cat\#7074, Cell Signaling Technology) and washed again. The membranes were treated with chemiluminescent reagents, and the protein bands were detected using the ChemiDoc XRS Plus System (Bio-Rad, Hercules, CA, USA). The membranes were stained with Coomassie blue to verify equal loading in all lanes. The band intensities were quantified using the Image Lab (Bio-Rad).

\section{Muscle protein synthesis}

In vivo rate of protein synthesis in the muscle was measured using the SUnSET method (25). Under isoflurane gas anesthesia, the mice were intraperitoneally injected with $0.04 \mu \mathrm{mol}$ puromycin/g body weight in sterile saline. The gastrocnemius muscle was removed $15 \mathrm{~min}$ after puromycin administration. Following homogenization as described above, and centrifugation at $2000 \times \mathrm{g}$ for $3 \mathrm{~min}$ at $4^{\circ} \mathrm{C}$, the supernatant was collected and processed for western blotting. Mouse monoclonal anti-puromycin antibody (cat\#MABE343, Millipore, Billerica, MA, USA) and anti-mouse IgG2a secondary antibody (\#115-035-206, Jackson ImmunoResearch, West Grove, PA, USA) were used to detect puromycin incorporation.

\section{Real-time PCR}

Total RNA was extracted from $10 \mathrm{mg}$ of powdered frozen muscle tissue using ISOGEN II (Nippon Gene, Tokyo, Japan). The purity and concentration of RNA were measured using a spectrophotometer (Synergy HT; BioTek, Winooski, VT, USA), and 500 ng of total RNA was reverse transcribed into cDNA using the PrimeScript ${ }^{\mathrm{TM}} \mathrm{RT}$ reagent Kit with gDNA Eraser (Takara Bio, Kusatsu, Japan). Realtime RT-qPCR was performed using the appropriate primer and TB Green ${ }^{\circledR}$ Premix Ex Taq ${ }^{\mathrm{TM}}$ II (Takara Bio) on a Thermal Cycler Dice ${ }^{\circledR}$ Real-Time System II (Takara Bio). Gene expression levels were measured by the absolute quantification method and were normalized to Gapdh. Primers used in the study were obtained from Thermo Fisher Scientific. Primer sequences are provided below: $c-M y c$ F-5'-CGGACACACAACGTCTTGGAA, R-5'-AGGATGTAGGCGGTGGCTTTT; 45S pre-rRNA F5'-CTGACACGCTGTCCTTTCCC-3', R- 5'-GTGAGCCGAaAtAagGTGGC-3'; Poll F- 5'- 
GGACTGGCAGAAGCATGGA-3', R- 5'-TCAAAGGGAGGTATGGCCTGC-3'; Ubf F- 5'AACCCAAAACTCCCCAGCAA-3', R- 5'-CCTCCTTCGTAGTGGCATCC-3'; Gapdh F- 5'TGCACCACCAACTGCTTAG-3', R- 5'-GGATGCAGGGATGATGTTC-3'

\section{Sequence library preparation, RNA-seq, and sequence data analysis}

RNA-seq was performed as previously described (26). Briefly, total RNA was extracted using TRIzol reagent according to the manufacturer's instructions. The extracted RNA was purified using the RNeasy Mini Kit (QIAGEN, Hilden, Germany) and RNA concentration was measured by spectrophotometry (Nanodrop One; Thermo Fisher Scientific). The RNA sequence library was prepared using the QuantSeq 3 mRNA-Seq Library Prep Kit (Lexogen). The prepared library was sequenced using MiniSeq (Illumina, San Diego, CA, USA) on MiniSeq system (Illumina). Quality checked and adapter sequence trimmed read count data were analyzed with integrated differential expression and pathway (iDEP) analysis (27). First, read count data were normalized using EdgeR (minimal counts per million: 0.5). Differential gene expression analyses were performed with limma-voom (c-Myc OE effect; false discovery rate (FDR) cutoff: 0.1, minimal fold change: 2) and DEseq2 (c-Myc OE $\times$ HiMC; FDR cutoff: 1, minimal fold change: 2). Pathway enrichment analyses were performed using the generally applicable gene set enrichment for pathway analysis (GAGE; FDR cutoff: 0.20) with the GO Biological Process. Raw data are available from Gene Expression Omnibus, accession\#GSE161402.

\section{Statistical analyses}

Data were analyzed by two-way ANOVA. Post-hoc analyses were performed using $t$-tests, with Benjamini and Hochberg FDR correction for multiple comparisons. The level of significance was set at $P<0.05$.

Data availability: Raw data of RNA-seq analysis are available from Gene Expression Omnibus, accession\#GSE161402. All remaining data are contained within the article. 


\section{Results}

\section{c-Myc overexpression stimulated ribosome biogenesis and muscle protein synthesis}

Two weeks of AAV6-c-Myc treatment in gastrocnemius muscle significantly increased the protein level of c-Myc about 3.5-fold (Fig. 1A and Table 1, main effect of AAV6-c-Myc treatment: P < 0.0001). The increased c-Myc expression was sufficient to increase protein synthesis by $\sim 20 \%$ and this was not affected by up to $9 \mathrm{~h}$ of fasting (Fig. 1B, main effect of AAV6-c-Myc treatment: $\mathrm{P}=0.0017$ ). Fasting decreased gastrocnemius muscle wet weight (main effect of fasting: $\mathrm{P}=0.0037$ ). Overexpression of $\mathrm{c}-$ Myc did not change the muscle wet weight within the time-frame studied (data not shown).

Total RNA and mRNA of POL1, UBTF, and 45S pre-rRNA were increased following c-Myc overexpression (Fig. 1C. main effect of AAV6-c-Myc treatment: $\mathrm{P}=0.0228,<0.0001,=0.0043$, and $<0.0001$, respectively). These effects were not influenced by up to $9 \mathrm{~h}$ of fasting, although $45 \mathrm{~S}$ prerRNA decreased with fasting time (main effect of fasting: $\mathrm{P}=0.0324$ ). On the other hand, although cMyc overexpression increased total 4E-BP1 (Fig. 1D and Table 1, main effect of AAV6-c-Myc treatment: $\mathrm{P}=0.0408$ ), it did not affect the decrease with fasting in phosphorylation of 4E-BP1 and p70S6K, downstream targets of mTORC1 (Fig. 1D and Table 1). Moreover, upstream regulators of mTORC1, including AMPK and REDD1, were unaffected following c-Myc overexpression, while REDD1 increased with fasting time (Fig. 1D and Table 1, main effect of fasting: $P=0.0062$ ). This indicates that c-Myc does not regulate mTORC1 signaling at rest or during acute fasting.

Apart from c-Myc, RNA-Seq identified 17,871 genes of which 16 genes were significantly altered (Up: 11 genes, Down: 5 genes) following AAV6-c-Myc treatment (Fig. 1E, raw data are available from Gene Expression Omnibus, accession\#GSE161402). GO pathway analysis revealed that c-Myc overexpression mainly upregulated genes related to ribosome biogenesis, ncRNA metabolic process, ncRNA processing, rRNA processing, rRNA metabolic process, ribosomal small subunit biogenesis, and ribonucleoprotein complex biogenesis, while it downregulated genes related to chemotaxis (Fig. $1 \mathrm{~F})$.

Fasting increased LC3-II (Fig. 1G, main effect of fasting: P = 0.0267), but did not affect LC3-I, p62 or general ubiquitination of proteins with no effect of c-Myc overexpression, indicating that c-Myc does not regulate resting or fasting-induced autophagy and the ubiquitin-proteasome system.

\section{c-Myc overexpression did not potentiate HiMC-induced muscle protein synthesis but increased the number of genes that were changed by HiMC}

AAV6-c-Myc treatment and HiMC individually and additively increased the c-Myc protein level (Fig. 2A and Table 2, main effect of AAV6-c-Myc treatment: $\mathrm{P}<0.0001$, main effect of HiMC: $\mathrm{P}=0.0004$ ). AAV6-c-Myc treatment tended to increase basal muscle protein synthesis (Fig. 2B, main effect of AAV6-c-Myc treatment: $\mathrm{P}=0.0985$ ) and HiMC increased muscle protein synthesis significantly (Fig. 
2B, main effect of HiMC: $\mathrm{P}=0$ 0.0036). However, although no interaction was observed (Fig. $2 \mathrm{~B}, \mathrm{P}=$ 0.1701), relative change in protein synthesis with HiMC was smaller in c-Myc overexpression group as compared with control group (Fig. 2B, $\mathrm{P}=0.041$ ).

Signaling-wise, HiMC increased the phosphorylation of p70S6K and 4E-BP1 (Fig. 2C and Table 2, main effect of HiMC: both $\mathrm{P}<0.0001$ ), with no significant changes in total proteins. However, although c-Myc overexpression did not affect HiMC-induced 4E-BP1 phosphorylation, it attenuated HiMCinduced p70S6K phosphorylation (Fig. 2C and Table 2, interaction: $\mathrm{P}=0.0091$ ). Neither HiMC nor cMyc overexpression affected the phosphorylation of AMPK (Fig. 2C and Table 2). HiMC decreased the expression of the endogenous mTORC1 inhibitor REDD1 independent of c-Myc overexpression (Fig. 2C and Table 2, main effect of HiMC: $\mathrm{P}<0.0001$ ).

RNA-seq identified 133 and 170 genes that were changed by HiMC in the control and c-Myc overexpressed group, respectively (Fig. 2E and 2G, raw data are available from Gene Expression Omnibus, accession\#GSE161402). Therefore, c-Myc overexpression increased the genes that were changed by HiMC. However, no significant differences were observed on any identified genes between the contracted muscles from the control and c-Myc overexpressing groups. 


\section{Discussion}

c-Myc is known to regulate several genes and stimulate ribosome biogenesis and protein synthesis in tumor cells $(14,15,22,23)$. Therefore, c-Myc upregulation in skeletal muscle following HiMC could be one of the mechanisms promoting HiMC-induced muscle anabolism. Here, we observed, for the first time, that c-Myc overexpression was sufficient to stimulate ribosome biogenesis and protein synthesis in skeletal muscles. Further, the number of genes that were regulated by HiMC was increased by c-Myc overexpression, indicating that c-Myc interacts with other transcriptional regulators activated by HiMC. Thus, our results suggest that c-Myc upregulation in skeletal muscle after HiMC contributes to HiMCinduced muscle anabolism.

As hypothesized, c-Myc overexpression stimulated ribosome biogenesis and protein synthesis in skeletal muscles. Given that HiMC increases c-Myc in skeletal muscles $(8,11)$, our results suggest that the HiMC-induced increase in c-Myc contributes to an increase in ribosome biogenesis and muscle protein synthesis following HiMC. mTORC1 is one of the major regulators of ribosome biogenesis and muscle protein synthesis $(8,11,28)$. In this study, c-Myc overexpression did not alter the phosphorylation of the downstream targets of mTORC1, p70S6K and 4E-BP1 in skeletal muscles. Therefore, our results indicate that c-Myc stimulates ribosome biogenesis and muscle protein synthesis independent of mTORC1. However, although c-Myc overexpression increased muscle protein synthesis, it did not increase muscle mass. In addition, neither autophagy nor ubiquitin-proteasome system was affected by c-Myc overexpression. Further studies, including detailed time-course studies, are required to identify the precise mechanism of c-Myc in the regulation of muscle mass.

While c-Myc overexpression stimulated ribosome biogenesis and muscle protein synthesis, it did not additively increase HiMC-induced muscle protein synthesis. In fact, the change in protein synthesis by HiMC was smaller in the c-Myc overexpression group. This indicated that upregulation of c-Myc genes is part of the mechanism behind HiMC-induced protein synthesis but that c-Myc-mediated ribosome biogenesis/increased translational capacity does not potentiate HiMC-induced muscle protein synthesis. Interestingly, c-Myc overexpression also inhibited HiMC-induced p70S6K phosphorylation, while no change was observed in upstream negative regulators of mTORC1, including AMPK and REDD1. The HiMC-induced increase in skeletal muscle c-Myc protein continues for a relatively long period (> 24 h) and is also observed under basal conditions after chronic HiMC (training) in rats and humans (8, 19, 20) along with an attenuated response of mTORC1/p70S6K signaling and muscle protein synthesis to HiMC following training (29-31). The mechanisms underlying the elevated resting protein synthesis and attenuated anabolic response to HiMC remain unclear. We speculate that c-Myc may mechanistically contribute to the chronically resistance exercise trained skeletal muscle phenotype, given that c-Myc overexpression mimics HiMC in terms of increased basal muscle protein synthesis 
but lowered mTORC1/p70S6K responsiveness to HiMC. Further studies are required to clarify this point.

c-Myc is a potent transcription factor that is estimated to regulate at least $15 \%$ of all genes (32). For example, c-Myc is highly expressed in cancer cells and is associated with the Warburg effect via transcriptional regulation of glycolytic enzymes (33). Therefore, we hypothesized that c-Myc overexpression would stimulate a wide range of genes, in particular those related to ribosome biogenesis- and glycolysis-related genes. However, in this study, RNA-Seq revealed that only 16 genes were altered following c-Myc overexpression, although c-Myc overexpression increased the changes in gene expression mediated by HiMC. Interestingly, a recent study identified 19,354 c-Myc-binding sites in chicken primary myoblasts, and only 1,061 in myotubes (21). These results indicate that while cMyc is still important for ribosome biogenesis and protein synthesis, it is quantitatively not a major transcription factor in fully differentiated muscle cells (adult skeletal muscle fibers in vivo).

\section{Conclusions}

We demonstrated that c-Myc overexpression stimulates ribosome biogenesis and protein synthesis without activation of mTORC1 in mouse skeletal muscle. However, it inhibits HiMC-induced phosphorylation of p70S6K and muscle protein synthesis, an effect reminiscent of chronic training conditions. Thus, c-Myc upregulation may both contribute to the HiMC-induced increase in protein synthesis and the decreased mTORC1/protein synthesis response observed with chronic resistance exercise training. 


\section{Declarations}

\section{Ethical approval}

All experimental procedures performed in this study were approved by the Danish Animal Experimental Inspectorate (License \# 2017-15-0201-01311).

\section{Consent for publication}

Not applicable.

\section{Data availability}

Raw data of RNA-seq analysis are available from Gene Expression Omnibus, accession\#GSE161402. All remaining data in this article are available from the corresponding author upon reasonable request.

\section{Conflicts of interest}

The authors declare that they have no conflicts of interest with the contents of this article.

\section{Funding}

This work was supported by Japan Society for the Promotion of Science KAKENHI Grant No. 26702028 and 19H03999 (to RO).

\section{Author's contributions}

TEJ and RO conceived and designed the work. TM, SA, JRK, CHO, ZL, KW, TS, KH, YT, and RO performed experiments. TM, SA, and KW analyzed the data. TM, SA, and RO interpreted the results of the experiments. RO drafted the manuscript. JRK, CHO, ZL, KN, TEJ, and RO edited and revised the manuscript. The authors read and approved the final manuscript 


\section{References}

1. Figueiredo VC. Revisiting the roles of protein synthesis during skeletal muscle hypertrophy induced by exercise. Am J Physiol Regul Integr Comp Physiol. 2019;317(5):R709-R18.

2. Goodman CA. Role of mTORC1 in mechanically induced increases in translation and skeletal muscle mass. J Appl Physiol (1985). 2019;127(2):581-90.

3. Figueiredo VC, McCarthy JJ. Regulation of Ribosome Biogenesis in Skeletal Muscle Hypertrophy. Physiology (Bethesda). 2019;34(1):30-42.

4. Glynn EL, Fry CS, Drummond MJ, Dreyer HC, Dhanani S, Volpi E, et al. Muscle protein breakdown has a minor role in the protein anabolic response to essential amino acid and carbohydrate intake following resistance exercise. Am J Physiol Regul Integr Comp Physiol. 2010;299(2):R533-40. 5. Phillips SM, Tipton KD, Aarsland A, Wolf SE, Wolfe RR. Mixed muscle protein synthesis and breakdown after resistance exercise in humans. Am J Physiol. 1997;273(1 Pt 1):E99-107.

6. Sabatini DM. Twenty-five years of mTOR: Uncovering the link from nutrients to growth. Proc Natl Acad Sci U S A. 2017;114(45):11818-25.

7. Saxton RA, Sabatini DM. mTOR Signaling in Growth, Metabolism, and Disease. Cell. 2017;169(2):361-71.

8. Ogasawara R, Fujita S, Hornberger TA, Kitaoka Y, Makanae Y, Nakazato K, et al. The role of mTOR signalling in the regulation of skeletal muscle mass in a rodent model of resistance exercise. Sci Rep. 2016;6:31142.

9. Ogasawara R, Jensen TE, Goodman CA, Hornberger TA. Resistance Exercise-Induced Hypertrophy: A Potential Role for Rapamycin-Insensitive mTOR. Exerc Sport Sci Rev. 2019;47(3):188-94.

10. Ogasawara R, Suginohara T. Rapamycin-insensitive mechanistic target of rapamycin regulates basal and resistance exercise-induced muscle protein synthesis. FASEB J. 2018:fj201701422R.

11. West DW, Baehr LM, Marcotte GR, Chason CM, Tolento L, Gomes AV, et al. Acute resistance exercise activates rapamycin-sensitive and -insensitive mechanisms that control translational activity and capacity in skeletal muscle. J Physiol. 2016;594(2):453-68.

12. You JS, McNally RM, Jacobs BL, Privett RE, Gundermann DM, Lin KH, et al. The role of raptor in the mechanical load-induced regulation of mTOR signaling, protein synthesis, and skeletal muscle hypertrophy. FASEB J. 2019;33(3):4021-34.

13. Philp A, Schenk S, Perez-Schindler J, Hamilton DL, Breen L, Laverone E, et al. Rapamycin does not prevent increases in myofibrillar or mitochondrial protein synthesis following endurance exercise. J Physiol. 2015;593(18):4275-84.

14. Ruggero D. The role of Myc-induced protein synthesis in cancer. Cancer Res. 2009;69(23):8839-43.

15. van Riggelen J, Yetil A, Felsher DW. MYC as a regulator of ribosome biogenesis and protein synthesis. Nat Rev Cancer. 2010;10(4):301-9.

16. Drummond MJ, Miyazaki M, Dreyer HC, Pennings B, Dhanani S, Volpi E, et al. Expression of growth-related genes in young and older human skeletal muscle following an acute stimulation of protein synthesis. J Appl Physiol (1985). 2009;106(4):1403-11.

17. Apro W, Wang L, Ponten M, Blomstrand E, Sahlin K. Resistance exercise induced mTORC1 signaling is not impaired by subsequent endurance exercise in human skeletal muscle. American journal of physiology Endocrinology and metabolism. 2013;305(1):E22-32.

18. Nader GA, von Walden F, Liu C, Lindvall J, Gutmann L, Pistilli EE, et al. Resistance exercise training modulates acute gene expression during human skeletal muscle hypertrophy. J Appl Physiol (1985). 2014;116(6):693-702.

19. Stec MJ, Kelly NA, Many GM, Windham ST, Tuggle SC, Bamman MM. Ribosome biogenesis may augment resistance training-induced myofiber hypertrophy and is required for myotube growth in vitro. American journal of physiology Endocrinology and metabolism. 2016;310(8):E652-E61.

20. Hammarstrom D, Ofsteng S, Koll L, Hanestadhaugen M, Hollan I, Apro W, et al. Benefits of higher resistance-training volume are related to ribosome biogenesis. J Physiol. 2020;598(3):543-65.

21. Luo W, Chen J, Li L, Ren X, Cheng T, Lu S, et al. c-Myc inhibits myoblast differentiation and promotes myoblast proliferation and muscle fibre hypertrophy by regulating the expression of its target genes, miRNAs and lincRNAs. Cell Death Differ. 2019;26(3):426-42. 
22. Morrish F, Hockenbery D. MYC and mitochondrial biogenesis. Cold Spring Harb Perspect Med. 2014;4(5).

23. Zeller KI, Zhao X, Lee CW, Chiu KP, Yao F, Yustein JT, et al. Global mapping of c-Myc binding sites and target gene networks in human B cells. Proc Natl Acad Sci U S A. 2006;103(47):17834-9.

24. Maruyama Y, Ikeda C, Wakabayashi K, Ato S, Ogasawara R. High-intensity muscle contraction-mediated increases in Akt1 and Akt2 phosphorylation do not contribute to mTORC1 activation and muscle protein synthesis. J Appl Physiol (1985). 2020.

25. Goodman CA, Mabrey DM, Frey JW, Miu MH, Schmidt EK, Pierre P, et al. Novel insights into the regulation of skeletal muscle protein synthesis as revealed by a new nonradioactive in vivo technique. FASEB J. 2011;25(3):1028-39.

26. Tamura Y, Kouzaki K, Kotani T, Nakazato K. Electrically stimulated contractile activityinduced transcriptomic responses and metabolic remodeling in $\mathrm{C} 2 \mathrm{C} 12$ myotubes: twitch vs. tetanic contractions. Am J Physiol Cell Physiol. 2020.

27. Ge SX, Son EW, Yao R. iDEP: an integrated web application for differential expression and pathway analysis of RNA-Seq data. BMC Bioinformatics. 2018;19(1):534.

28. Marabita M, Baraldo M, Solagna F, Ceelen JJM, Sartori R, Nolte H, et al. S6K1 Is Required for Increasing Skeletal Muscle Force during Hypertrophy. Cell Rep. 2016;17(2):501-13.

29. Ogasawara R, Kobayashi K, Tsutaki A, Lee K, Abe T, Fujita S, et al. mTOR signaling response to resistance exercise is altered by chronic resistance training and detraining in skeletal muscle. J Appl Physiol (1985). 2013;114(7):934-40.

30. Brook MS, Wilkinson DJ, Mitchell WK, Lund JN, Szewczyk NJ, Greenhaff PL, et al. Skeletal muscle hypertrophy adaptations predominate in the early stages of resistance exercise training, matching deuterium oxide-derived measures of muscle protein synthesis and mechanistic target of rapamycin complex 1 signaling. FASEB J. 2015;29(11):4485-96.

31. Ato S, Tsushima D, Isono Y, Suginohara T, Maruyama Y, Nakazato K, et al. The Effect of Changing the Contraction Mode During Resistance Training on mTORC1 Signaling and Muscle Protein Synthesis. Front Physiol. 2019;10:406.

32. Dang CV, O'Donnell KA, Zeller KI, Nguyen T, Osthus RC, Li F. The c-Myc target gene network. Semin Cancer Biol. 2006;16(4):253-64.

33. Osthus RC, Shim H, Kim S, Li Q, Reddy R, Mukherjee M, et al. Deregulation of glucose transporter 1 and glycolytic gene expression by c-Myc. J Biol Chem. 2000;275(29):21797-800. 
Table 1. Effect of c-Myc overexpression and fasting on total signaling proteins.

\begin{tabular}{|c|c|c|c|c|c|c|}
\hline & \multicolumn{2}{|c|}{$0 \mathrm{~h}$} & \multicolumn{2}{|c|}{$3 \mathrm{~h}$} & \multicolumn{2}{|c|}{$9 \mathrm{~h}$} \\
\hline & $\mathrm{CON}$ & Myc & $\mathrm{CON}$ & Myc & $\mathrm{CON}$ & Myc \\
\hline c-Myc* & $1.00 \pm 0.55$ & $3.90 \pm 2.24$ & $0.67 \pm 0.48$ & $3.22 \pm 1.69$ & $0.78 \pm 0.40$ & $3.72 \pm 2.13$ \\
\hline p-p70S6K $\mathrm{K}^{\#}$ & $1.00 \pm 0.39$ & $0.97 \pm 0.41$ & $0.17 \pm 0.06$ & $0.21 \pm 0.09$ & $0.17 \pm 0.04$ & $0.14 \pm 0.02$ \\
\hline p70S6K & $1.00 \pm 0.20$ & $0.92 \pm 0.18$ & $0.69 \pm 0.09$ & $0.89 \pm 0.20$ & $0.81 \pm 0.13$ & $0.92 \pm 0.14$ \\
\hline $\mathrm{p}-4 \mathrm{E}-\mathrm{BP} 1^{\#}$ & $1.00 \pm 0.17$ & $1.02 \pm 0.10$ & $0.75 \pm 0.07$ & $0.75 \pm 0.09$ & $0.67 \pm 0.07$ & $0.81 \pm 0.10$ \\
\hline$\gamma /$ total $4 \mathrm{E}-\mathrm{BP} 1^{\#}$ & $1.00 \pm 0.15$ & $0.97 \pm 0.16$ & $0.76 \pm 0.16$ & $0.79 \pm 0.16$ & $0.72 \pm 0.09$ & $0.66 \pm 0.13$ \\
\hline $4 \mathrm{E}-\mathrm{BP} 1^{*}$ & $1.00 \pm 0.38$ & $1.10 \pm 0.34$ & $0.86 \pm 0.09$ & $1.17 \pm 0.29$ & $0.94 \pm 0.34$ & $1.00 \pm 0.40$ \\
\hline REDD1 $^{\#}$ & $1.00 \pm 0.26$ & $1.19 \pm 0.19$ & $1.04 \pm 0.32$ & $1.05 \pm 0.40$ & $1.76 \pm 0.53$ & $1.81 \pm 0.41$ \\
\hline p-AMPK & $1.00 \pm 0.12$ & $1.02 \pm 0.11$ & $1.02 \pm 0.09$ & $1.00 \pm 0.13$ & $1.05 \pm 0.14$ & $1.00 \pm 0.11$ \\
\hline AMPK & $1.00 \pm 0.27$ & $0.94 \pm 0.27$ & $0.94 \pm 0.21$ & $1.05 \pm 0.11$ & $0.96 \pm 0.26$ & $0.89 \pm 0.37$ \\
\hline LC3-I & $1.00 \pm 0.41$ & $1.09 \pm 0.35$ & $1.10 \pm 0.30$ & $1.28 \pm 0.37$ & $0.99 \pm 0.28$ & $1.00 \pm 0.27$ \\
\hline LC3-II ${ }^{\#}$ & $1.00 \pm 0.38$ & $1.30 \pm 0.76$ & $2.44 \pm 1.24$ & $2.11 \pm 0.64$ & $2.59 \pm 0.99$ & $2.74 \pm 1.08$ \\
\hline LC3-II/I ${ }^{\#}$ & $1.00 \pm 0.44$ & $1.08 \pm 0.48$ & $1.95 \pm 0.73$ & $1.58 \pm 0.50$ & $2.34 \pm 0.47$ & $2.52 \pm 0.71$ \\
\hline p62 & $1.00 \pm 0.17$ & $0.94 \pm 0.24$ & $0.71 \pm 0.14$ & $0.90 \pm 0.24$ & $0.81 \pm 0.15$ & $0.77 \pm 0.15$ \\
\hline Ubiquitin & $1.00 \pm 0.09$ & $1.03 \pm 0.10$ & $0.93 \pm 0.08$ & $0.91 \pm 0.16$ & $0.86 \pm 0.17$ & $0.92 \pm 0.18$ \\
\hline
\end{tabular}

Data are expressed as means \pm SD. *Main effect of Myc overexpression, ${ }^{*}$ main effect of fasting. 
Table 2. Effect of c-Myc overexpression and HiMC on total signaling proteins.

\begin{tabular}{ccccc}
\hline & \multicolumn{2}{c}{ CON } & \multicolumn{2}{c}{ Myc } \\
\cline { 2 - 5 } HiMC $^{*}$ & - & + & - & + \\
\cline { 2 - 5 } c-Myc*\# $^{*}$ & $1.00 \pm 0.41$ & $1.87 \pm 0.29$ & $2.65 \pm 0.61$ & $5.17 \pm 2.04$ \\
p70S6K & $1.00 \pm 0.19$ & $0.99 \pm 0.19$ & $1.10 \pm 0.18$ & $1.02 \pm 0.22$ \\
$\gamma /$ total 4E-BP1 ${ }^{\#}$ & $1.00 \pm 0.28$ & $1.75 \pm 0.39$ & $0.99 \pm 0.28$ & $1.50 \pm 0.90$ \\
4E-BP1 & $1.00 \pm 0.28$ & $0.90 \pm 0.24$ & $1.06 \pm 0.40$ & $0.90 \pm 0.38$ \\
REDD1 & $1.00 \pm 0.37$ & $0.52 \pm 0.23$ & $0.79 \pm 0.29$ & $0.47 \pm 0.25$ \\
p-AMPK & $1.00 \pm 0.30$ & $0.86 \pm 0.20$ & $1.00 \pm 0.21$ & $1.08 \pm 0.54$ \\
AMPK & $1.00 \pm 0.40$ & $0.93 \pm 0.33$ & $0.91 \pm 0.29$ & $0.66 \pm 0.30$ \\
\hline Data are & & & & \\
\end{tabular}

Data are expressed as means $\pm \mathrm{SD}$. *Main effect of Myc overexpression, ${ }^{\#}$ main effect of HiMC. 


\section{Figures}

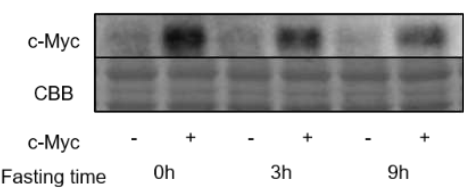

B

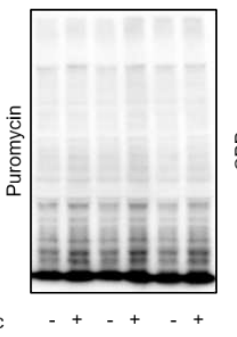

c-Myc $\quad-+\cdots+-+$

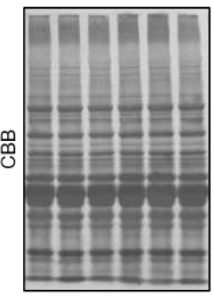

$-++\cdots$ Oh $3 \mathrm{~h} \quad 9 \mathrm{~h}$

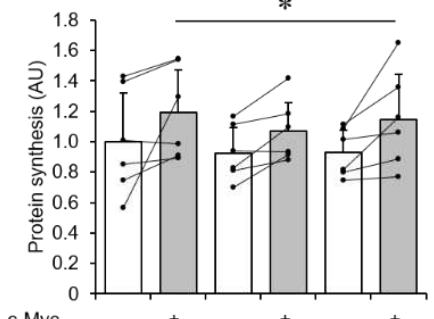

c-Myc Fasting time
$9 \mathrm{~h}$

D

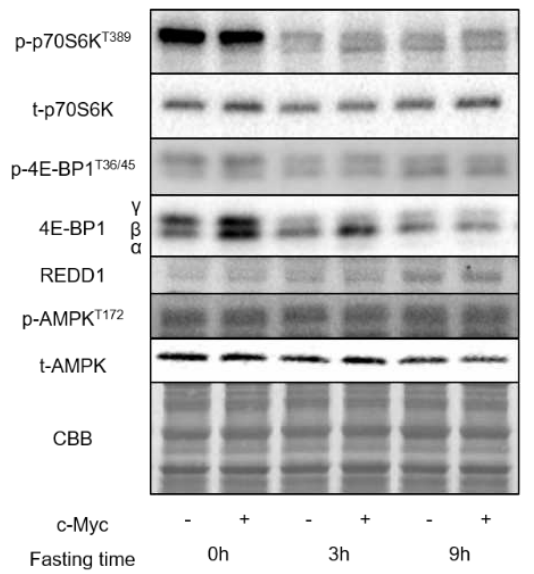

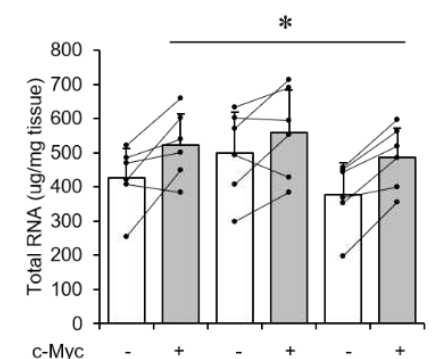

Fasting time $\quad$ oh $\quad 3 \mathrm{~h} \quad 9 \mathrm{~h} \quad$ Fasting time $\quad$ oh $\quad 3 \mathrm{~h} \quad 9 \mathrm{~h}$

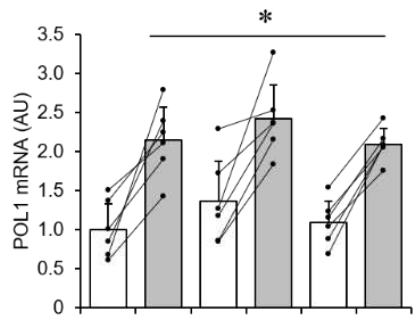

c-Myc Fasting time
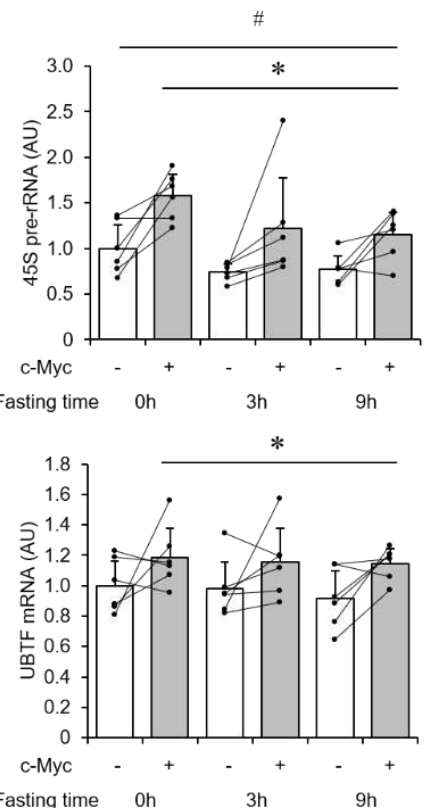

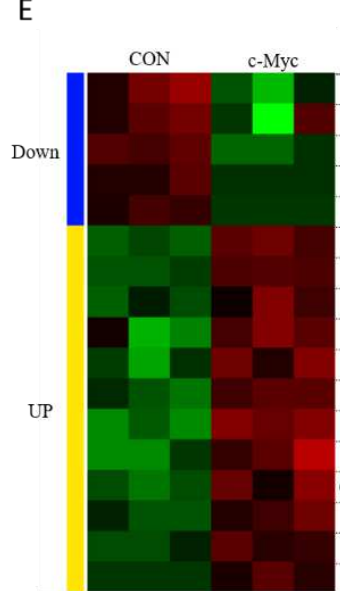

F

\begin{tabular}{|ccc} 
Symbol & $\begin{array}{c}\text { log2 Fold } \\
\text { Change }\end{array}$ & Adj.Pval \\
\hline Mfap4 & -1.59 & $3.00 \mathrm{E}-02$ \\
\hline Cdknlc & -1.76 & $2.03 \mathrm{E}-03$ \\
\hline Mthfs & -2.54 & $5.35 \mathrm{E}-02$ \\
\hline Gm3839 & -2.57 & $4.30 \mathrm{E}-02$ \\
\hline Penk & -3.02 & $1.42 \mathrm{E}-03$ \\
\hline Gnl3 & 1.04 & $7.26 \mathrm{E}-02$ \\
\hline Nme1 & 1.11 & $5.99 \mathrm{E}-04$ \\
\hline Timm10 & 1.29 & $1.62 \mathrm{E}-04$ \\
\hline Pstk & 1.37 & $6.70 \mathrm{E}-02$ \\
\hline Pycr1 & 1.64 & $4.19 \mathrm{E}-02$ \\
\hline Sh3bp1 & 1.88 & $2.73 \mathrm{E}-02$ \\
\hline Slc25a22 & 2.60 & $3.58 \mathrm{E}-02$ \\
\hline Particl & 2.66 & $2.46 \mathrm{E}-02$ \\
\hline Gm10638 & 2.69 & $2.81 \mathrm{E}-02$ \\
\hline Hpdl & 2.79 & $1.70 \mathrm{E}-02$ \\
\hline Arhgdig & 3.44 & $1.92 \mathrm{E}-04$ \\
\hline Myc & 3.86 & $1.60 \mathrm{E}-06$ \\
\hline & & \\
\hline
\end{tabular}

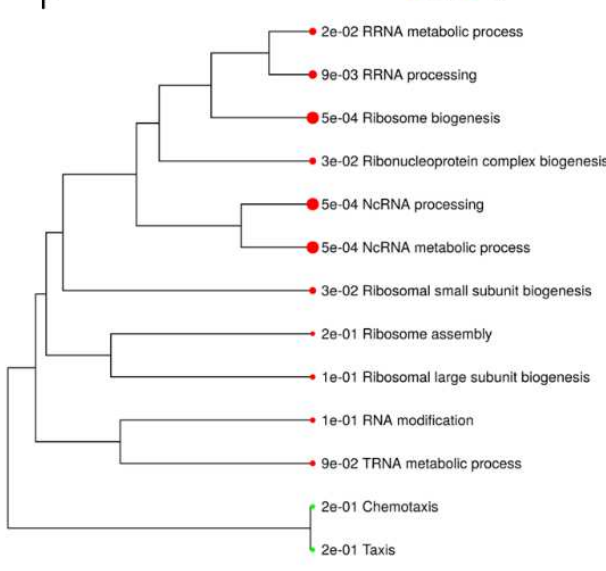

G

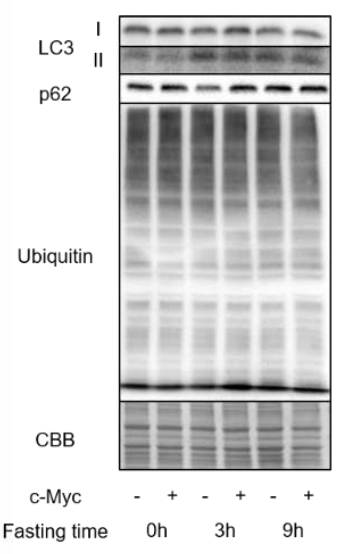

Figure 1. Effect of c-Myc overexpression and fasting. AAV6-c-Myc treatment increased c-Myc protein expression $(\mathrm{A}, \mathrm{n}=6)$. c-Myc overexpression increased protein synthesis $(\mathrm{B}, \mathrm{n}=6)$ and ribosome biogenesis $(C, n=6)$, but not mTORC1 signaling $(D, n=6)$. RNA-Seq identified 16 genes that were significantly altered by c-Myc overexpression $(\mathrm{E}, \mathrm{n}=3)$ and subsequent $\mathrm{GO}$ pathway analysis revealed 13 biological processes that were modified by c-Myc overexpression (F). c-Myc overexpression did not affect autophagy and ubiquitin-proteasome system $(G, n=6)$. Data are expressed as means \pm standard deviation. $* P<0.05$ main effect of c-Myc overexpression. 
A

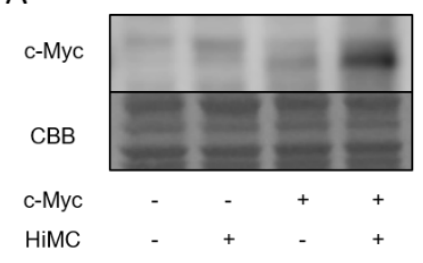

C

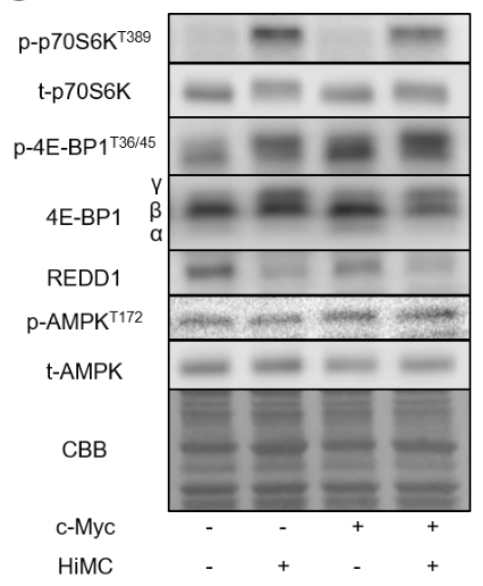

B

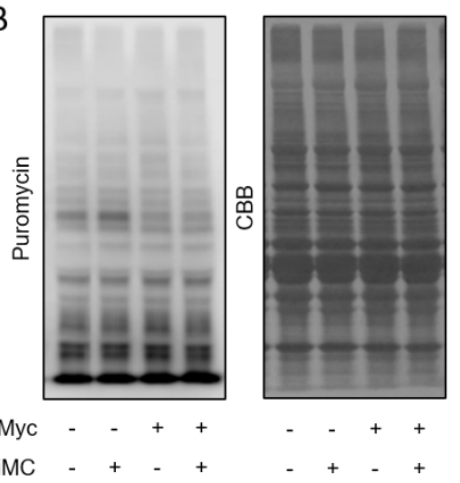

D

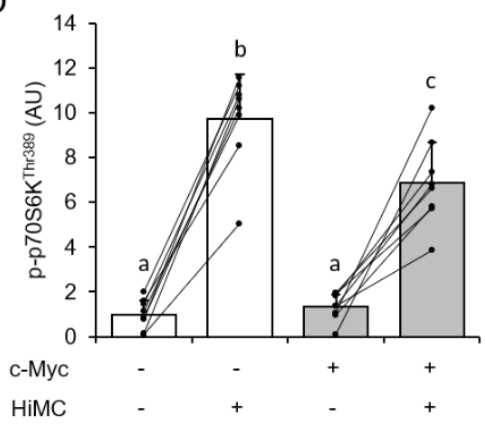

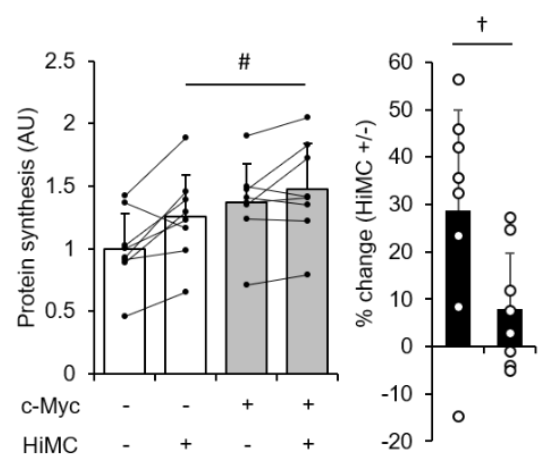

HiMC - + ++

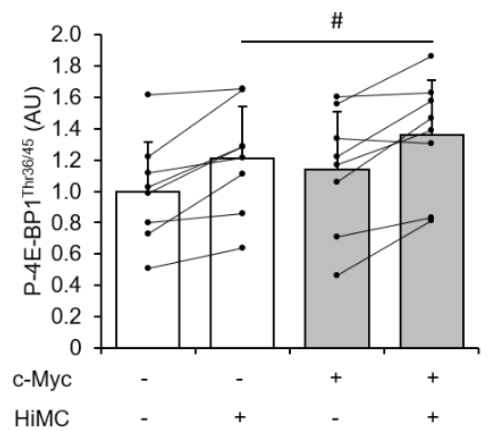

\section{Control group}

E

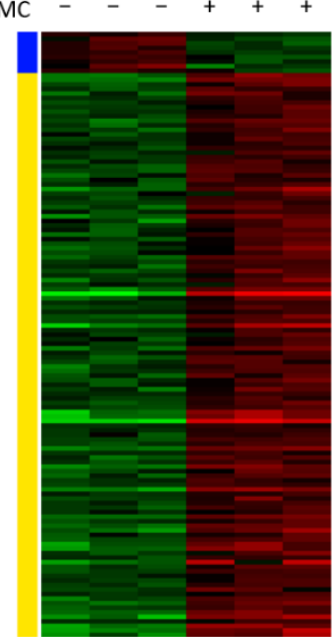

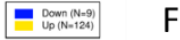

- Up

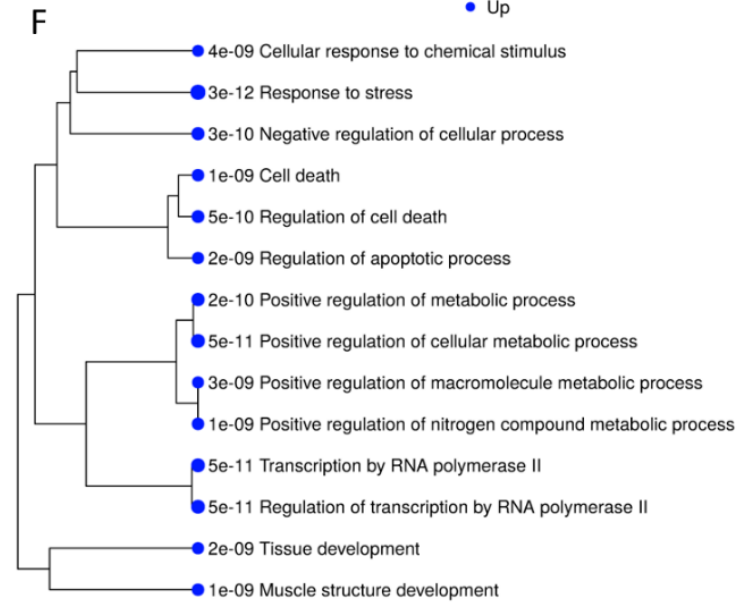

c-Myc overexpression group

G HiMC

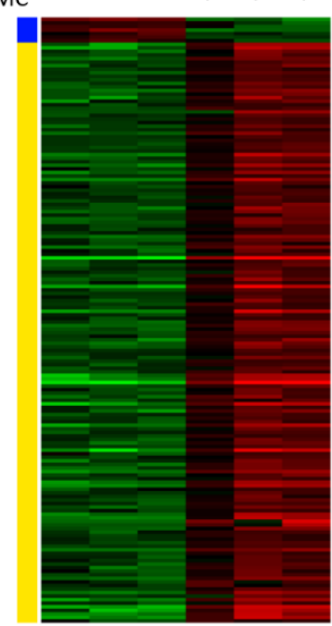

H

- Up
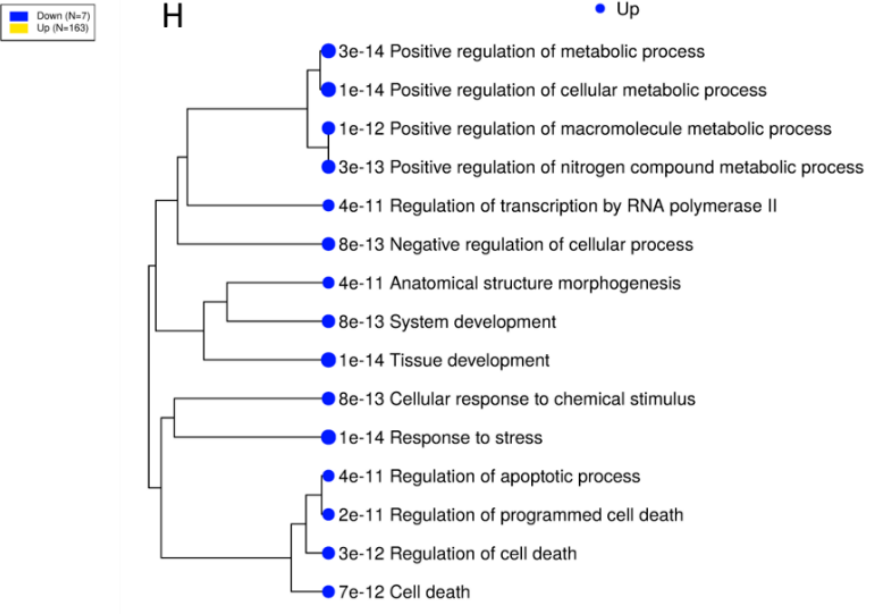
Figure 2. Effect of c-Myc overexpression and high-intensity muscle contraction (HiMC). AAV6-c-Myc treatment and HiMC additively increased c-Myc protein expression $(\mathrm{A}, \mathrm{n}=8)$. HiMC increased muscle protein synthesis but relative change in protein synthesis by HiMC was greater in the control group (B, $\mathrm{n}=8$ ). c-Myc overexpression did not affect HiMC-induced mTORC1 signaling except for p70S6K phosphorylation ( $\mathrm{C}$ and $\mathrm{D}, \mathrm{n}=8)$. RNA-seq identified 133 and 170 genes that were changed by HiMC in the control and c-Myc overexpression groups, respectively ( $\mathrm{E}$ and $\mathrm{G}, \mathrm{n}=3$ ), and subsequent $\mathrm{GO}$ pathway analysis revealed 14 and 15 biological processes that were modified by HiMC in the control and c-Myc overexpression groups, respectively ( $\mathrm{F}$ and $\mathrm{H})$. Data are expressed as means \pm standard deviation. Different characters indicate significant differences $(\mathrm{p}<0.05),{ }^{\#} P<0.05$ main effect of HiMC, ${ }^{\dagger} P<0.05$ vs. CON. 
A

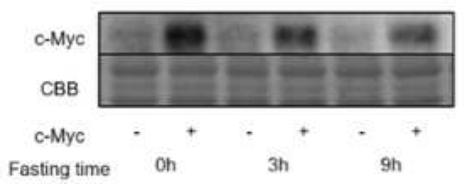

B

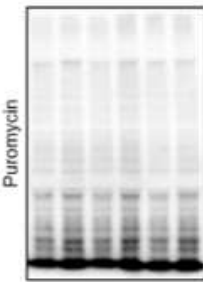

c-Myc .....

Fasting time on $3 \mathrm{~h}$ 9h
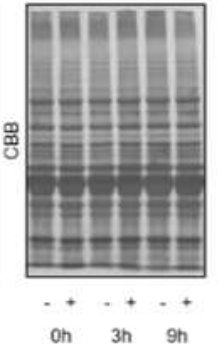

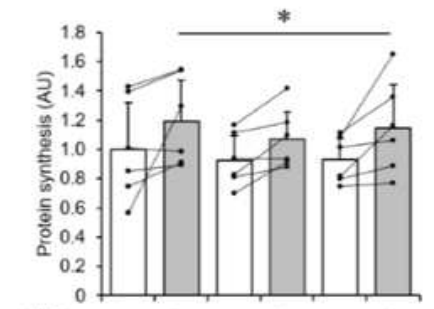

c-Myc Fasting time

9 h
C

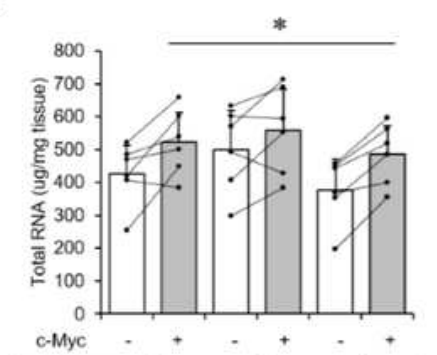

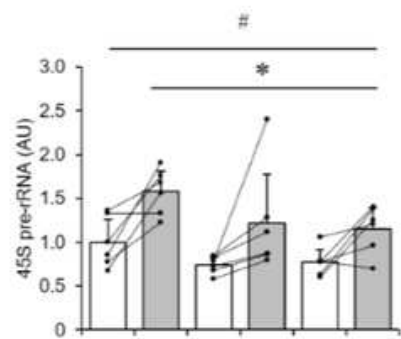

c-Myc

Fasting time on $3 \mathrm{~h} \quad 9 \mathrm{~h}$ Fasting time on $3 \mathrm{~h}$ 9h

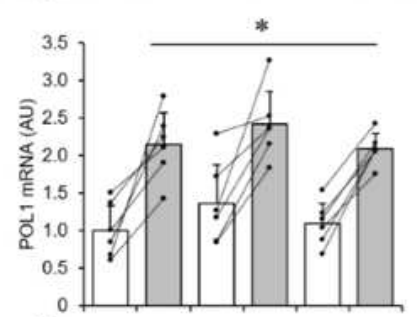

Myc

Fasting time 0

E

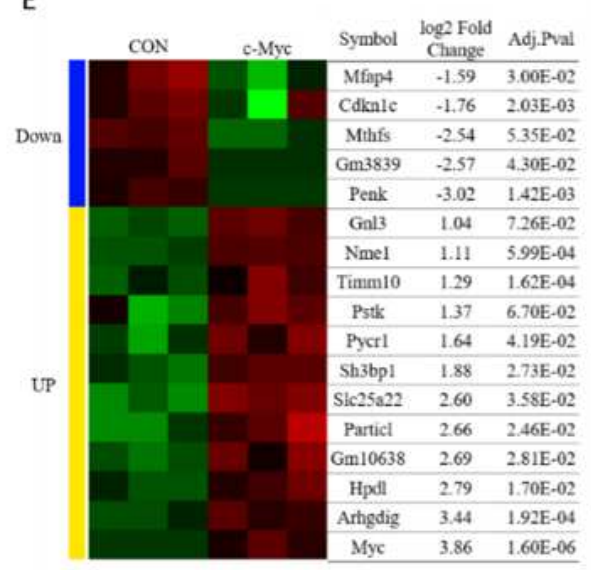

F

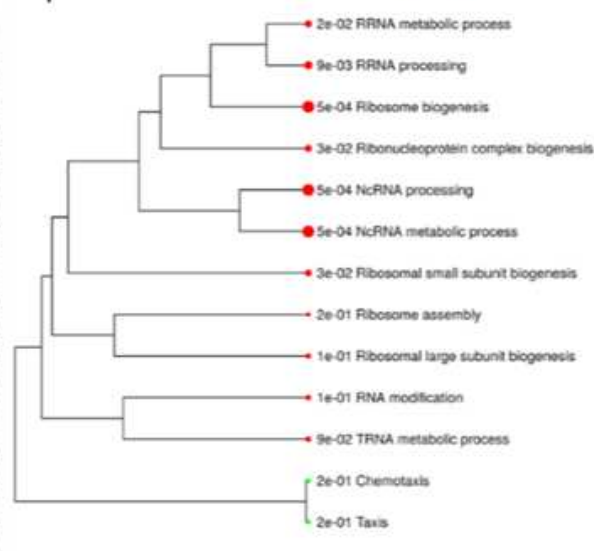

D

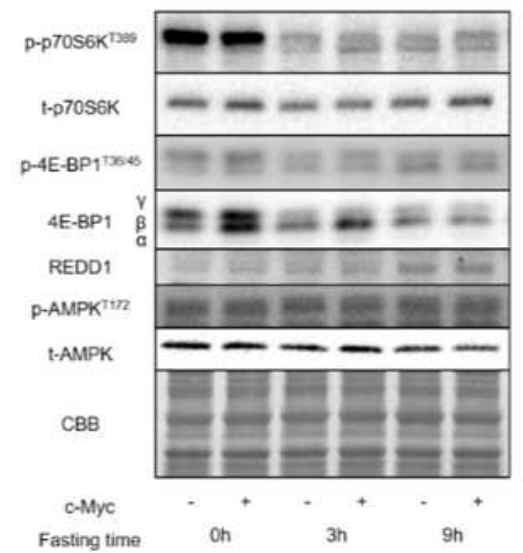

G

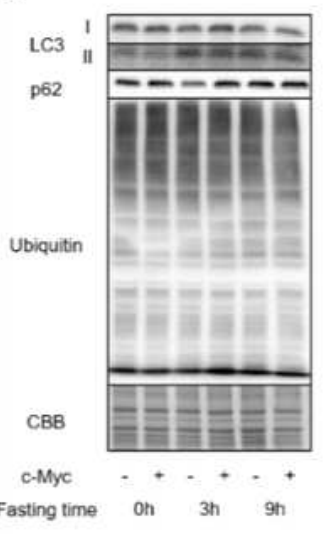

Figure 1

Effect of c-Myc overexpression and fasting. AAV6-c-Myc treatment increased c-Myc protein expression (A, $n=6)$. $c-M y c$ overexpression increased protein synthesis $(B, n=6)$ and ribosome biogenesis $(C, n=6)$, but not mTORC1 signaling $(D, n=6)$. RNA-Seq identified 16 genes that were significantly altered by c-Myc 
overexpression ( $E, n=3)$ and subsequent $G 0$ pathway analysis revealed 13 biological processes that were modified by c-Myc overexpression (F). c-Myc overexpression did not affect autophagy and ubiquitinproteasome system $(G, n=6)$. Data are expressed as means \pm standard deviation. ${ }^{*} P<0.05$ main effect of c-Myc overexpression.
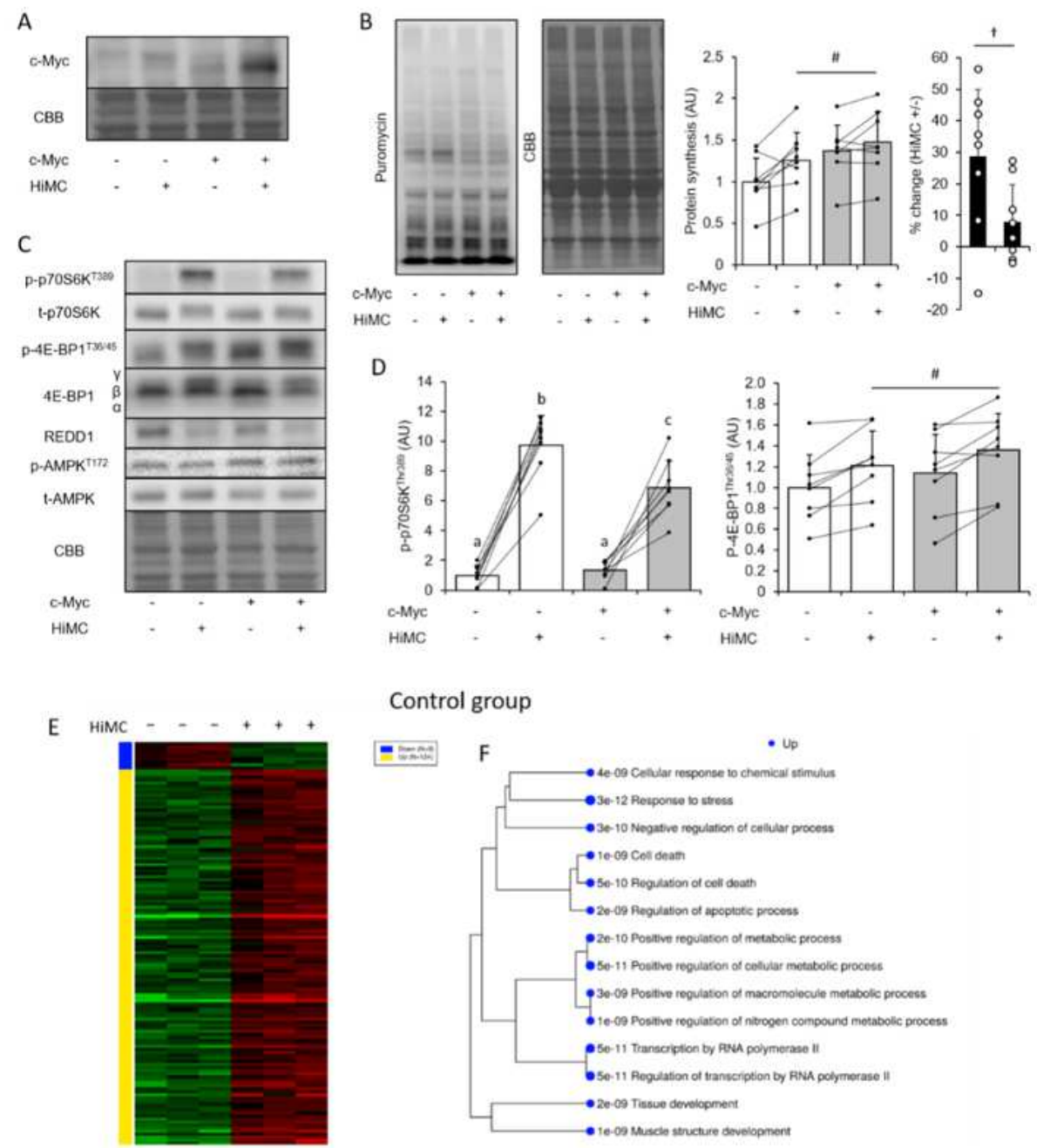

Control group

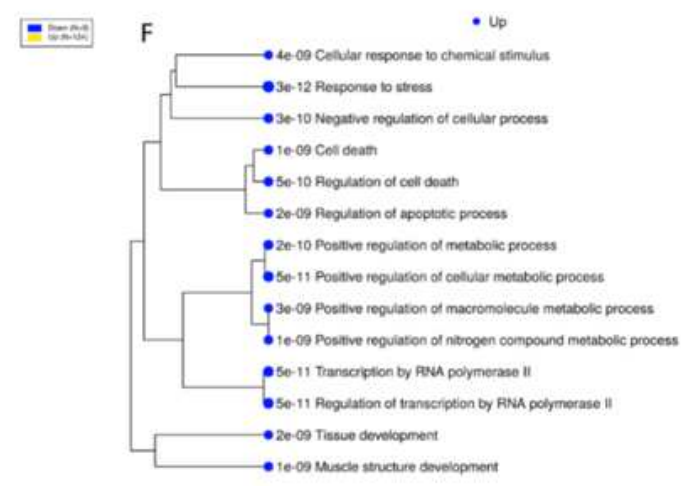

c-Myc overexpression group

G HiMC - - - + + +
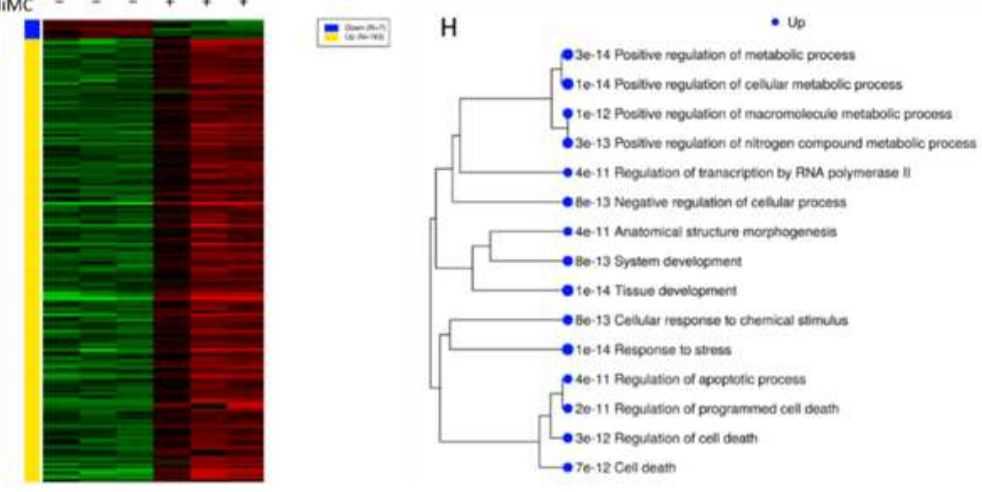

Figure 2 
Effect of c-Myc overexpression and high-intensity muscle contraction (HiMC). AAV6-c-Myc treatment and HiMC additively increased $c-M y c$ protein expression $(A, n=8)$. HiMC increased muscle protein synthesis but relative change in protein synthesis by HiMC was greater in the control group $(B, n=8)$. c-Myc overexpression did not affect HiMC-induced mTORC1 signaling except for p70S6K phosphorylation (C and $D, n=8)$. RNA-seq identified 133 and 170 genes that were changed by HiMC in the control and c-Myc overexpression groups, respectively $(E$ and $G, n=3)$, and subsequent $G 0$ pathway analysis revealed 14 and 15 biological processes that were modified by HiMC in the control and c-Myc overexpression groups, respectively $(\mathrm{F}$ and $\mathrm{H})$. Data are expressed as means \pm standard deviation. Different characters indicate significant differences $(p<0.05)$, \#P< 0.05 main effect of HiMC, $+P<0.05$ vs. CON. 